\title{
Correction to: Hand dexterity, not handgrip strength, is associated with executive function in Japanese community-dwelling older adults: a cross-sectional study
}

Kimi Estela Kobayashi-Cuya ${ }^{1,2}$, Ryota Sakurai ${ }^{1 *}$, Naoko Sakuma ${ }^{1}$, Hiroyuki Suzuki ${ }^{1}$, Masashi Yasunaga ${ }^{1}$, Susumu Ogawa', Toru Takebayashi ${ }^{2}$ and Yoshinori Fujiwara' ${ }^{1}$

\section{Correction}

Following the publication of this article [1], the authors reported a typesetting error in Table 1 that resulted in missing asterisks. This correction shows both the incorrect and correct versions of Table 1 , and Table 1 has also been corrected in the original article.

The incorrect version is:

Table 1 Comparison of covariates, hand and cognitive variables among age categories $(N=326)$

\begin{tabular}{|c|c|c|c|c|c|}
\hline \multirow[t]{2}{*}{ Variables } & \multicolumn{4}{|l|}{ Age categories } & \multirow[t]{2}{*}{$p$-value } \\
\hline & $60-64(n=54)$ & $65-69(n=104)$ & $70-74(n=101)$ & $\geq 75(n=67)$ & \\
\hline Years of education & $14.4 \pm 2.5$ & $13.4 \pm 2.2$ & $13.3 \pm 2.5$ & $12.8 \pm 2.7$ & 0.004 \\
\hline TMIG-IC & $12.2 \pm 0.8$ & $12.3 \pm 0.9$ & $12.4 \pm 0.9$ & $12.2 \pm 1.3$ & 0.645 \\
\hline GDS & $2.3 \pm 2.1$ & $3.1 \pm 2.5$ & $2.5 \pm 2.3$ & $3.0 \pm 2.3$ & 0.09 \\
\hline Heart disease, $n(\%)$ & $2(3.7)$ & $1(1.0)$ & $11(10.9)$ & $7(10.4)$ & $0.012^{\mathrm{a}}$ \\
\hline Diabetes, $n(\%)$ & $2(3.7)$ & $9(8.7)$ & $6(5.9)$ & $5(7.5)$ & $0.670^{\mathrm{a}}$ \\
\hline Hypertension, $n(\%)$ & $11(20.4)$ & $23(22.1)$ & $27(26.7)$ & $28(41.8)$ & $0.020^{\mathrm{a}}$ \\
\hline Stroke, $n(\%)$ & $3(5.6)$ & $6(5.8)$ & $5(5.0)$ & $3(4.5)$ & $0.983^{\mathrm{a}}$ \\
\hline \multicolumn{6}{|c|}{ Hand motor variables, mean \pm SD } \\
\hline Handgrip strength $(\mathrm{Kg})$ & $23.4 \pm 4.7$ & $22.6 \pm 5.3$ & $22.9 \pm 7.0$ & $20.9 \pm 6.3$ & 0.104 \\
\hline PPT (Number of pegs) & $14.5 \pm 1.5$ & $13.7 \pm 2.1$ & $13.1 \pm 1.9$ & $11.9 \pm 1.9^{\dagger, \neq}$ & $<0.001$ \\
\hline \multicolumn{6}{|c|}{ Cognitive variables, mean $\pm \mathrm{SD}$} \\
\hline MMSE & $29.2 \pm 0.9$ & $28.8 \pm 1.3$ & $28.9 \pm 1.0$ & $28.6 \pm 1.3$ & 0.023 \\
\hline TMT-A & $30.2 \pm 7.7$ & $35.3 \pm 13.2$ & $37.9 \pm 11.1$ & $43.2 \pm 12.4^{\dagger, \neq}$ & $<0.001$ \\
\hline TMT-B & $74.5 \pm 18.6$ & $91.9 \pm 37.6$ & $107.4 \pm 39.9^{\dagger}$ & $121.6 \pm 40.8^{\dagger}$ & $<0.001$ \\
\hline Digit symbol ${ }^{b}$ & $67.7 \pm 9.3$ & $60.2 \pm 13.8$ & $55.3 \pm 10.9$ & $45.6 \pm 8.4^{+, \neq}$ & $<0.001$ \\
\hline
\end{tabular}

Values are expressed as mean \pm SD

TMIG-IC Tokyo Metropolitan Institute of Gerontology - Index of Competence, GDS Geriatric Depression Scale, MMSE Mini-Mental State Examination, TMT Trail Making Test Bonferroni correction for post-hoc tests: ${ }^{*} p<0.008$ vs. $60-64{ }^{\dagger} p<0.008$ vs. $65-69 ;{ }^{\ddagger} p<0.008$ vs. $70-74$

${ }^{a}$ The Chi-square test was performed

${ }^{\mathrm{b}}$ The total number of subjects analyzed was 207

* Correspondence: r_sakurai@hotmail.co.jp

${ }^{1}$ Research Team for Social Participation and Community Health, Tokyo

Metropolitan Institute of Gerontology, 35-2 Sakae-cho, Itabashi-ku, Tokyo

173-0015, Japan

Full list of author information is available at the end of the article

(c) The Author(s). 2018 Open Access This article is distributed under the terms of the Creative Commons Attribution 4.0 International License (http://creativecommons.org/licenses/by/4.0/), which permits unrestricted use, distribution, and reproduction in any medium, provided you give appropriate credit to the original author(s) and the source, provide a link to the Creative Commons license, and indicate if changes were made. The Creative Commons Public Domain Dedication waiver (http://creativecommons.org/publicdomain/zero/1.0/) applies to the data made available in this article, unless otherwise stated. 
The correct version is:

Table 1 Comparison of covariates, hand and cognitive variables among age categories $(N=326)$

\begin{tabular}{|c|c|c|c|c|c|}
\hline \multirow[t]{2}{*}{ Variables } & \multicolumn{4}{|c|}{ Age categories } & \multirow[t]{2}{*}{$p$-value } \\
\hline & $\begin{array}{l}60-64 \\
(n=54)\end{array}$ & $\begin{array}{l}65-69 \\
(n=104)\end{array}$ & $\begin{array}{l}70-74 \\
(n=101)\end{array}$ & $\begin{array}{l}\geq 75 \\
(n=67)\end{array}$ & \\
\hline Years of education & $14.4 \pm 2.5$ & $13.4 \pm 2.2$ & $13.3 \pm 2.5^{*}$ & $12.8 \pm 2.7^{*}$ & 0.004 \\
\hline TMIG-IC & $12.2 \pm 0.8$ & $12.3 \pm 0.9$ & $12.4 \pm 0.9$ & $12.2 \pm 1.3$ & 0.645 \\
\hline GDS & $2.3 \pm 2.1$ & $3.1 \pm 2.5$ & $2.5 \pm 2.3$ & $3.0 \pm 2.3$ & 0.09 \\
\hline Heart disease, $n(\%)$ & $2(3.7)$ & $1(1.0)$ & $11(10.9)$ & $7(10.4)$ & $0.012^{\mathrm{a}}$ \\
\hline Diabetes, $n(\%)$ & $2(3.7)$ & $9(8.7)$ & $6(5.9)$ & $5(7.5)$ & $0.670^{a}$ \\
\hline Hypertension, n (\%) & $11(20.4)$ & $23(22.1)$ & $27(26.7)$ & $28(41.8)$ & $0.020^{\mathrm{a}}$ \\
\hline Stroke, $n(\%)$ & $3(5.6)$ & $6(5.8)$ & $5(5.0)$ & $3(4.5)$ & $0.983^{\mathrm{a}}$ \\
\hline \multicolumn{6}{|c|}{ Hand motor variables, mean \pm SD } \\
\hline Handgrip strength $(\mathrm{Kg})$ & $23.4 \pm 4.7$ & $22.6 \pm 5.3$ & $22.9 \pm 7.0$ & $20.9 \pm 6.3$ & 0.104 \\
\hline PPT (Number of pegs) & $14.5 \pm 1.5$ & $13.7 \pm 2.1$ & $13.1 \pm 1.9^{*}$ & $11.9 \pm 1.9^{*, t, \neq}$ & $<0.001$ \\
\hline \multicolumn{6}{|c|}{ Cognitive variables, mean \pm SD } \\
\hline MMSE & $29.2 \pm 0.9$ & $28.8 \pm 1.3$ & $28.9 \pm 1.0$ & $28.6 \pm 1.3^{*}$ & 0.023 \\
\hline TMT-A & $30.2 \pm 7.7$ & $35.3 \pm 13.2$ & $37.9 \pm 11.1^{*}$ & $43.2 \pm 12.4^{*, t, \neq}$ & $<0.001$ \\
\hline TMT-B & $74.5 \pm 18.6$ & $91.9 \pm 37.6^{*}$ & $107.4 \pm 39.9^{*, t}$ & $121.6 \pm 40.8^{*,+}$ & $<0.001$ \\
\hline Digit symbol ${ }^{b}$ & $67.7 \pm 9.3$ & $60.2 \pm 13.8$ & $55.3 \pm 10.9^{*}$ & $45.6 \pm 8.4^{*, t, \neq}$ & $<0.001$ \\
\hline
\end{tabular}

Values are expressed as mean \pm SD

TMIG-IC Tokyo Metropolitan Institute of Gerontology - Index of Competence, GDS Geriatric Depression Scale, MMSE Mini-Mental State Examination, TMT Trail Making Test

Bonferroni correction for post-hoc tests: ${ }^{*} p<0.008$ vs. $60-64 ;{ }^{\dagger} p<0.008$ vs. $65-69 ;{ }^{\ddagger} p<0.008$ vs. $70-74$

${ }^{\text {a } T h e ~ C h i-s q u a r e ~ t e s t ~ w a s ~ p e r f o r m e d ~}$

${ }^{\text {b}}$ The total number of subjects analyzed was 207

\section{Author details}

${ }^{1}$ Research Team for Social Participation and Community Health, Tokyo Metropolitan Institute of Gerontology, 35-2 Sakae-cho, Itabashi-ku, Tokyo 173-0015, Japan. ${ }^{2}$ Department of Preventive Medicine and Public Health, School of Medicine, Keio University, 35 Shinanomachi, Shinjuku-ku, Tokyo 160-8582, Japan.

Received: 3 September 2018 Accepted: 4 September 2018

Published online: 11 September 2018

\section{Reference}

1. Kobayashi-Cuya et al. BMC Geriatr (2018) 18:192 https://doi.org/10.1186/ s12877-018-0880-6 